\title{
Adaptive-Gain Second-Order Sliding Mode Control of Attitude Tracking of Flexible Spacecraft
}

\author{
Chutiphon Pukdeboon \\ Nonlinear Dynamic Analysis Research Center, Department of Mathematics, Faculty of Applied Science, \\ King Mongkut's University of Technology North Bangkok, Bangkok 10800, Thailand
}

Correspondence should be addressed to Chutiphon Pukdeboon; cpd@kmutnb.ac.th

Received 15 March 2014; Accepted 11 April 2014; Published 21 May 2014

Academic Editor: Her-Terng Yau

Copyright ( $(2014$ Chutiphon Pukdeboon. This is an open access article distributed under the Creative Commons Attribution License, which permits unrestricted use, distribution, and reproduction in any medium, provided the original work is properly cited.

\begin{abstract}
This paper investigates the robust finite-time control problem for flexible spacecraft attitude tracking maneuver in the presence of model uncertainties and external disturbances. Two robust attitude tracking controllers based on finite-time second-order sliding mode control algorithms are presented to solve this problem. For the first controller, a novel second-order sliding mode control scheme is developed to achieve high-precision tracking performance. For the second control law, an adaptive-gain second-order sliding mode control algorithm combing an adaptive law with second-order sliding mode control strategy is designed to relax the requirement of prior knowledge of the bound of the system uncertainties. The rigorous proofs show that the proposed controllers provide finite-time convergence of the attitude and angular velocity tracking errors. Numerical simulations on attitude tracking control are presented to demonstrate the performance of the developed controllers.
\end{abstract}

\section{Introduction}

In recent years, considerable attention has been focused on spacecraft attitude control problems. Attitude control systems are required to offer the present generation of spacecraft with attitude maneuver, tracking, and pointing capabilities. Since the attitude dynamics of spacecraft is coupled and highly nonlinear, the attitude controller designs are usually difficult. Besides, the effect of motion of the elastic appendages makes the control problem more complicated. In the absence of external disturbances, the modal-independent proportionalderivative (PD) controller proposed in [1] can achieve asymptotical stability of both the attitude and angular velocity. In practical situations, the model parameters of the spacecraft may not be exactly known and the spacecraft is always subject to external disturbances. Thus, the attitude control problem with uncertainties and external disturbance has also attracted a great deal of attention. Various nonlinear robust control approaches [2-4] have been proposed for solving the attitude tracking control problem of flexible spacecraft. These control schemes include adaptive control $[5,6]$, sliding mode control
$[7,8]$, output feedback control $[9,10]$, optimal control [11, 12], and intelligent control [13].

Among these methods, sliding mode control (SMC) has been shown to be a potential approach when applied to a system with disturbances which satisfy the matched uncertainty condition [14]. Robust attitude controllers of flexible spacecraft based on the SMC scheme have been proposed in $[15,16]$. These control laws can achieve global asymptotic stability and provide good tracking results. However, these controllers were designed based on an asymptotic stability analysis which implies that the system trajectories converge to the equilibrium with infinite settling time. It is well known that finite-time stabilization of dynamical systems may provide a faster disturbance attenuation besides giving faster convergence to the required orientation. A recently developed technique for finite-time stabilization is the terminal sliding mode (TSM) method $[17,18]$ which can be used to design a controller that will guarantee a finite-time convergence to the origin. In $[19,20]$, the attitude motion of flexible spacecraft has been studied and the TSM method was used to design finite- time controllers. 
However, the TSM method usually provides lower tracking precision when compared with second-order sliding mode control (SOSMC) schemes $[21,22]$. This technique preserves the robustness ability of SMC and also yields improved accuracy and performance. Various real-life applications have been controlled in a practical implementation of SOSMC schemes (e.g., see [23-25]). The SOSMC strategies have been successfully applied to attitude tracking controller designs for a rigid spacecraft in $[26,27]$, but these schemes have been rarely used for attitude tracking control of flexible spacecraft.

In this paper, by virtue of SOSMC designs, the proposed attitude tracking control laws can guarantee the convergence of attitude tracking errors in finite-time. First, a novel finitetime SOSMC scheme is designed to achieve fast and accurate tracking responses. Next, to obtain the second control law, we combine the adaptive law with the SOSMC scheme proposed in [27]. This controller relaxes the requirement of prior knowledge on the bound of uncertainties. With the time scaling approach [28], the control parameters can be tuned by using only one variable.

The main contributions of this paper are as follows.

(I) Robust finite-time control algorithms based on SOSMC schemes have been rarely studied for attitude tracking maneuver of a flexible spacecraft. The finitetime stability of the proposed control laws is analyzed using Lyapunov stability concepts.

(II) A novel adaptive law for the gains of the SOSMC algorithm has been rarely developed by using the time scaling approach. The presented control method does not need a priori knowledge of the uncertainty and disturbance bounds.

This paper is organized as follows. In Section 2, the dynamic and kinematic equations governing the attitude model $[29,30]$ are described and the control design problem is formulated. Section 3 presents a novel SOSMC control algorithm for a flexible spacecraft. The sliding manifold is chosen and the sliding control law is studied and a proof of finite-time convergence of this controller is given. Section 4 proposes an adaptive-gain SOSMC law. The stability of the closed-loop system is analyzed. A numerical example of spacecraft tracking maneuvers is presented in Section 5 to verify the usefulness of the proposed controllers. In Section 6 , we present conclusions.

\section{Nonlinear Mode and Problem Formulation}

2.1. Spacecraft Attitude Dynamics and Kinematics. The unit quaternion is adopted to describe the attitude of the spacecraft for global representation without singularities [29]. The unit quaternion $Q$ is defined by

$$
Q=\left[\begin{array}{c}
n \sin \left(\frac{\phi}{2}\right) \\
\cos \left(\frac{\phi}{2}\right)
\end{array}\right]=\left[\begin{array}{c}
q \\
q_{4}
\end{array}\right],
$$

where $n \in \mathscr{R}^{3}$ is a unit vector called the Euler axis, $\phi \in \mathscr{R}$ denotes the magnitude of Euler axis rotation and $q \in \mathscr{R}^{3}$ and $q_{4} \in \mathscr{R}$ are the vector components and the scalar of the unit quaternion, respectively. They are subject to the constraint $q^{T} q+q_{4}^{2}=1$. Consider the first time derivative of $Q$. The kinematic equations are given by $[29,30]$

$$
\begin{gathered}
\dot{q}=\frac{1}{2}\left(q_{4} I_{3}+q^{\times}\right) \omega, \\
\dot{q}_{4}=-\frac{1}{2} q^{T} \omega,
\end{gathered}
$$

where $I_{3}$ is a $3 \times 3$ identity matrix, and $q^{\times}$is a skew-symmetric matrix:

$$
q^{\times}=\left[\begin{array}{ccc}
0 & -q_{3} & q_{2} \\
q_{3} & 0 & -q_{1} \\
-q_{2} & q_{1} & 0
\end{array}\right] .
$$

2.2. Relative Attitude Error Kinematics. We explain briefly the attitude error using quaternions. We define here the desired quaternion $Q_{r}=\left[\begin{array}{ll}q_{r}^{T} & q_{4 r}\end{array}\right]^{T} \in \mathscr{R}^{3} \times \mathscr{R}$ with $q_{r}=$ $\left[\begin{array}{lll}q_{1 r} & q_{2 r} & q_{3 r}\end{array}\right]^{T}$. Also the attitude error $Q_{e}=\left[\begin{array}{ll}q_{e}^{T} & q_{4 e}\end{array}\right]^{T} \in$ $\mathscr{R}^{3} \times \mathscr{R}$ with $q_{e}=\left[\begin{array}{lll}q_{1 e} & q_{2 e} & q_{3 e}\end{array}\right]^{T}$. Using the quaternion multiplication law, we obtain

$$
Q_{e}=\left[\begin{array}{c}
q_{4 r} q-q_{4} q_{r}-q_{r}^{\times} q \\
q_{4} q_{4 r}+q^{T} q_{r}
\end{array}\right]
$$

subject to the constraint

$$
Q_{e}^{T} Q_{e}=\left(q^{T} q+q_{4}^{2}\right)\left(q_{r}^{T} q_{r}+q_{4 r}^{2}\right)=1
$$

The kinematic equation for the attitude error is expressed as $[29,30]$

$$
\dot{Q}_{e}=\frac{1}{2}\left[\begin{array}{c}
q_{e}^{\times}+q_{4 e} I_{3 \times 3} \\
-q_{e}^{T}
\end{array}\right] \omega_{e} \text {. }
$$

2.3. Flexible Spacecraft Dynamics. The equation governing a flexible spacecraft is expressed as [3]

$$
\begin{gathered}
J \dot{\omega}+\delta^{T} \ddot{\eta}=-\omega^{\times}\left(J \omega+\delta^{T} \dot{\eta}\right)+u+d, \\
\ddot{\eta}+\bar{C} \dot{\eta}+\bar{K} \eta=-\delta \dot{\omega},
\end{gathered}
$$

where $J=J^{T} \in \mathscr{R}^{3 \times 3}$ is the total inertia matrix of the spacecraft, $\eta \in \mathscr{R}^{4}$ is the modal displacement, and $\delta \in \mathscr{R}^{4 \times 3}$ is the coupling matrix between the central rigid body and the flexible attachments. $\omega=\left[\begin{array}{lll}\omega_{1} & \omega_{2} & \omega_{3}\end{array}\right]^{T} \in \mathscr{R}^{3}$ represents the angular velocity vector and $\omega^{\times}$is a skew-symmetric matrix with a formula similar to $q^{\times} \cdot u=\left[\begin{array}{lll}u_{1} & u_{2} & u_{3}\end{array}\right]^{T} \in \mathscr{R}^{3}$ is the control input, $d=\left[\begin{array}{lll}d_{1} & d_{2} & d_{3}\end{array}\right]^{T} \in \mathscr{R}^{3}$ represents the external disturbance torque, and $\bar{K}$ and $\bar{C}$ denote the stiffness and damping matrices, respectively, which are defined as

$$
\begin{aligned}
& \bar{K}=\operatorname{diag}\left(\omega_{n i}^{2}, i=1,2, \ldots, N\right), \\
& \bar{C}=\operatorname{diag}\left(2 \zeta_{i} \omega_{n i}, i=1,2, \ldots, N\right)
\end{aligned}
$$

with damping $\zeta_{i}$ and natural frequency $\omega_{n i}$. 
If terms $\delta^{T} \ddot{\eta}$ and $\omega^{\times} \delta^{T} \dot{\eta}$ are considered as lumped perturbations to rigid body dynamics, (7) can be obtained as

$$
J \dot{\omega}=-\omega^{\times} J \omega+u+d+\Delta f(\dot{\eta}, \ddot{\eta}, \omega),
$$

where $\Delta f(\dot{\eta}, \ddot{\eta}, \omega)=-\delta^{T} \ddot{\eta}-\omega^{\times} \delta^{T} \dot{\eta}$ may be considered as the lumped perturbation. Let the angular velocity error be defined as $\omega_{e}=\omega-\omega_{d}$; then the error dynamics are given by

$$
J \dot{\omega}_{e}=-\left(\omega_{e}+\omega_{d}\right)^{\times} J\left(\omega_{e}+\omega_{d}\right)-J \dot{\omega}_{d}+u+T_{d},
$$

where $T_{d}=d+\Delta f(\dot{\eta}, \ddot{\eta}, \omega)$.

Using (6), one has

$$
\dot{q}_{e}=\Xi\left(q_{e}, q_{4 e}\right) \omega_{e}
$$

with $\Xi\left(q_{e}, q_{4 e}\right)=(1 / 2)\left(q_{e}^{\times}+q_{4 e} I_{3}\right)$.

Define $P \triangleq \Xi^{-1}$ and $J^{*} \triangleq P^{T} J P$ and consider the time derivative of (11). One can obtain [3]

$$
J^{*} \ddot{q}_{e}+C^{*} \dot{q}_{e}+N^{*}=\tau_{u}+\tau_{d},
$$

where

$$
\begin{gathered}
C^{*}=-J^{*} \dot{P}^{-1} P-2 P^{T}\left(J P \dot{q}_{e}\right)^{\times} P, \\
\tau_{u}=\frac{1}{2} P^{T} u, \quad \tau_{d}=\frac{1}{2} P^{T} T_{d}, \\
N^{*}=P^{T}\left[\left(P \dot{q}_{e}\right)^{\times} J \omega_{d}\right]+P^{T}\left[\left(\omega_{d}\right)^{\times} J P \dot{q}_{e}\right] \\
+\frac{1}{2} P^{T}\left[\left(\omega_{d}\right)^{\times} J \omega_{d}\right]+\frac{1}{2} P^{T} J \dot{\omega}_{d} .
\end{gathered}
$$

Throughout the remainder of this paper, the following are assumed.

Assumption 1. The elastic oscillation and its rate are supposed to be bounded; that is to say, $\|\eta(t)\|$ and $\|\dot{\eta}(t)\|$ are bounded during the whole attitude tracking process.

Let $x_{1}=q_{e}$ and $x_{2}=\dot{q}_{e}$; the spacecraft model (12) can be written as

$$
\begin{gathered}
\dot{x}_{1}=x_{2}, \\
\dot{x}_{2}=F+B_{0} u+\widetilde{d},
\end{gathered}
$$

where $F=-J^{*}\left(C^{*} x_{2}+N^{*}\right), B_{0}=(1 / 2)\left(P^{T} J P\right)^{-1} P^{T}$, and $\widetilde{d}=\left(P^{T} J P\right)^{-1} \tau_{d}$.

Assumption 2. The first time derivative of the disturbance vector $\tilde{d}$ to the spacecraft system in (12) is assumed to be bounded and it satisfies the following condition:

$$
\left|\dot{\vec{d}}_{i}(t)\right| \leq \varrho,
$$

where $\varrho$ is a positive constant.
2.4. Problem Statement. In this work, we consider tracking maneuvers. The control objective is to realize desired rotations of flexible spacecraft in the presence of external disturbances. In other words, we shall find a controller $u$ subject to (14) such that, for all initial conditions, the desired rotations are achieved as follows:

$$
\lim _{t \rightarrow T} q_{e}=0, \quad \lim _{t \rightarrow T} q_{4 e}=1, \quad \lim _{t \rightarrow T} \omega_{e}=0,
$$

where $T$ is a positive constant. Note that, when $q_{e} \rightarrow 0$, we have $q_{4 e} \rightarrow 1$, due to the constraint relation (5).

2.5. Finite-Time Stability. We now restate the concepts related to finite-time stability $[31,32]$.

Definition 3 (see [31]). Consider a time invariant system in the form of

$$
\dot{x}=f(x), \quad f(0)=0, \quad x \in \mathscr{R}^{n},
$$

where $f: \widehat{U}_{0} \rightarrow \mathscr{R}^{n}$ is continuous on an open neighborhood $\widehat{U}_{0}$ of the origin. The equilibrium $x=0$ of the system (17) is (locally) finite-time stable if (i) it is Lyapunov stable, in $\widehat{U}$, an open neighborhood of the origin, with $\widehat{U} \subset \widehat{U}_{0}$, (ii) it is finitetime convergent in $\widehat{U}$, that is, for any condition $x_{0} \in \widehat{U} \backslash\{0\}$, there is a settling time $T>0$, such that every solution $x\left(t, x_{0}\right)$ of the system (17) is defined with $x\left(t, x_{0}\right) \in \widehat{U}\{0\}$ for $t \in[0, T]$ and it satisfies

$$
\lim _{t \rightarrow T\left(x_{0}\right)} x\left(t, x_{0}\right)=0
$$

and $x\left(t, x_{0}\right)=0$ if $t \geq T$. Moreover, if $\widehat{U}=\mathscr{R}^{n}$, the origin is globally finite-time stable.

Definition 4. Consider a controlled system

$$
\dot{x}=f(x)+g(x) u, \quad x \in \mathscr{R}^{n}, u \in \mathscr{R}^{m}
$$

with $g(x) \neq 0$. It is finite-time stabilizable if there is a feedback law $u(x)$ such that $x=0$ is a (locally) finite-time stable equilibrium of closed-loop system.

Lemma 5 (see [32]). Suppose that the origin is a finite-time stable equilibrium of (17) and the settling time function $T_{f}$ is continuous at zero, where $f(\cdot)$ is continuous. Let $\widehat{U}$ be defined as in Definition 3 and let $\iota \in(0,1)$. There exists a continuous scalar function $V$ such that (i) $V$ is positive definite and (ii) $\dot{V}$ is real valued and continuous on $\widehat{U}$ and there exists $c \in \mathscr{R}^{+}$ such that

$$
\dot{V}+c V^{\iota} \leq 0, \quad \text { for any } \iota \in(0,1) .
$$

Lemma 6 (Feng et al. [18]). For any numbers $\lambda_{1}>0, \lambda_{2}>0$, and $0<\omega<1$, an extended Lyapunov contion of finite-time stability can be given in the form of fast terminal sliding mode as

$$
\dot{V}(x)+\lambda_{1} V(x)+\lambda_{2} V_{2}^{\infty}(x) \leq 0,
$$

where the settling time can be estimated by

$$
T_{r} \leq \frac{1}{\lambda_{1}(1-\omega)} \ln \frac{\lambda_{1} V^{1-\Phi}\left(x_{0}\right)+\lambda_{2}}{\lambda_{2}} .
$$




\section{SOSMC Algorithm}

In this section, a novel SOSMC algorithm is proposed to accomplish attitude tracking control of a flexible spacecraft. Under Assumptions 1 and 2, this control scheme is designed such that the attitude tracking error $q_{e}$ and the angular velocity error $\omega_{e}$ approach zero in finite time although uncertainties and external disturbances are taken into account. This new SOSMC law is achieved by a Lyapunov function approach, so the finite-time stability of the closed-loop system of the spacecraft model (14) is ensured. The goal is to enforce the sliding mode on the manifold

$$
S=x_{2}+\kappa x_{1}
$$

where $S=\left[\begin{array}{lll}S_{1} & S_{2} & S_{3}\end{array}\right]^{T} \in \mathscr{R}^{3}$ and $\kappa$ is a positive constant. From (23), the first time derivative of $S$ is obtained as

$$
\begin{aligned}
\dot{S} & =\dot{x}_{2}+\kappa \dot{x}_{1}, \\
& =F+B_{0} u+\tilde{d}+\kappa x_{2} .
\end{aligned}
$$

Then, it is followed by choosing a SOSMC law to force the system trajectory onto the sliding surface. Let $s_{1}=S$ and $s_{2}=$ $\dot{S}$; the proposed control law is defined as

$$
\begin{aligned}
u=\left(B_{0}\right)^{-1}\left(-F-\kappa x_{2}-\mu_{1} \operatorname{sign}^{\alpha}\left(s_{1}\right)-\mu_{2} s_{1}\right. \\
\left.\quad-\int_{0}^{t}\left(\mu_{3} \operatorname{sign}^{\alpha}\left(s_{2}(\tau)\right)+\mu_{4} s_{2}(\tau)\right) d \tau\right),
\end{aligned}
$$

where $0<\alpha<1$. The gain matrices $\mu_{1}=\operatorname{diag}\left(\mu_{11}, \mu_{12}, \mu_{13}\right)$, $\mu_{2}=\operatorname{diag}\left(\mu_{21}, \mu_{22}, \mu_{23}\right), \mu_{3}=\operatorname{diag}\left(\mu_{31}, \mu_{32}, \mu_{33}\right)$, and $\mu_{4}=$ $\operatorname{diag}\left(\mu_{41}, \mu_{42}, \mu_{43}\right)$, with $\mu_{1 i}, \mu_{2 i}, \mu_{3 i}$ and $\mu_{4 i}(i=1,2,3)$, are positive gains. The function $\operatorname{sign}^{\gamma}(y)$ is defined as

$$
\begin{aligned}
& \operatorname{sign}^{\gamma}(y) \\
& \quad=\left[\left|y_{1}\right|^{\gamma} \operatorname{sign}\left(y_{1}\right)\left|y_{2}\right|^{\gamma} \operatorname{sign}\left(y_{2}\right) \cdots\left|y_{m}\right|^{\gamma} \operatorname{sign}\left(y_{m}\right)\right]^{T}
\end{aligned}
$$

with $0<\gamma<1$ and $y \in \mathscr{R}^{m}$.

Substituting (25) into (24), the closed-loop dynamics is obtained as

$$
\begin{aligned}
\dot{s_{1}}= & \mu_{1} \operatorname{sign}^{\alpha}\left(s_{1}\right)-\mu_{2} s_{1} \\
& -\int_{0}^{t}\left(\mu_{3} \operatorname{sign}^{\alpha}\left(s_{2}(\tau)\right)+\mu_{4} s_{2}(\tau)\right) d \tau+\widetilde{d} .
\end{aligned}
$$

Let $\dot{\tilde{d}}_{i}(t)=\delta_{i}(t), z_{1 i}=s_{1 i}$ and $z_{2 i}=-\int_{0}^{t}\left(\mu_{3 i} \operatorname{sign}^{\alpha}\left(s_{2 i}(\tau)\right)-\right.$ $\left.\mu_{4 i} s_{2 i}(\tau)\right) d \tau+\tilde{d}_{i}$. The system (27) can be written in scalar form $(i=1,2,3)$ as

$$
\begin{array}{r}
\dot{z}_{1 i}=-\mu_{1 i}\left|z_{1 i}\right|^{\alpha} \operatorname{sign}\left(z_{1 i}\right)+z_{2 i}-\mu_{2 i} z_{1 i}, \\
\dot{z}_{2 i}=-\mu_{3 i}\left|z_{2 i}\right|^{\alpha} \operatorname{sign}\left(z_{2 i}\right)-z_{2 i}-\mu_{4 i} z_{1 i}+\delta_{i} .
\end{array}
$$

Next, the proof of finite-time convergence to the origin is given.
Theorem 7. Under Assumptions 1 and 2 and the action of the control law (25), the state trajectories $z_{1 i}$ and $z_{2 i}$ converge in finite time to the region

$$
\begin{array}{r}
\|\varepsilon\| \leq \Delta=\min \left\{\Delta_{1}, \Delta_{2}\right\}, \\
\Delta_{1}=\frac{2 \varrho}{\epsilon_{2}}, \quad \Delta_{2}=\sqrt{2}\left(\frac{\sqrt{2} \varrho}{\epsilon_{1}}\right)^{1 / \alpha},
\end{array}
$$

where $\varepsilon=\left[\begin{array}{ll}z_{1 i} & z_{2 i}\end{array}\right]^{T}$ and $\Delta_{1}, \Delta_{2}>0$ are the results from the chosen gains.

Proof. We choose the Lyapunov function $V_{1}=(1 / 2) \varepsilon^{2}$ for analyzing the closed-loop system dynamics of (28). The time derivative of $V_{1}$ is given by

$$
\begin{aligned}
\dot{V}_{1}= & z_{1 i}\left(-\mu_{1 i}\left|z_{1 i}\right|^{\alpha} \operatorname{sign}\left(z_{1 i}\right)+z_{2 i}-\mu_{2 i} z_{1 i}\right) \\
& +z_{2 i}\left(-\mu_{3 i}\left|z_{2 i}\right|^{\alpha} \operatorname{sign}\left(z_{2 i}\right)-z_{1 i}-\mu_{4 i} z_{2 i}+\delta_{i}\right) \\
= & -\mu_{1 i}\left|z_{1 i}\right|^{\alpha+1}-\mu_{2 i} z_{1 i}^{2}-\mu_{3 i}\left|z_{2 i}\right|^{\alpha+1}-\mu_{4 i} z_{2 i}^{2}+\delta_{i} z_{2 p}
\end{aligned}
$$

which can be further written as

$$
\begin{aligned}
\dot{V}_{1} \leq & -\mu_{1 m}\left|z_{1 i}\right|^{\alpha+1}-\mu_{2 m} z_{1 i}^{2}-\mu_{3 m}\left|z_{2 i}\right|^{\alpha+1} \\
& -\mu_{4 m} z_{2 i}^{2}+\varrho\left|z_{2 i}\right|
\end{aligned}
$$

where $\mu_{1 m}=\min \left(\mu_{1 i}\right), \mu_{2 m}=\min \left(\mu_{2 i}\right), \mu_{3 m}=\min \left(\mu_{3 i}\right)$, and $\mu_{4 m}=\min \left(\mu_{4 i}\right), i=1,2,3$.

Letting $\bar{\mu}_{1}=\min \left(\mu_{1 m}, \mu_{3 m}\right)$ and $\bar{\mu}_{2}=\min \left(\mu_{2 m}, \mu_{4 m}\right)$, one obtains

$$
\begin{array}{r}
\dot{V}_{1} \leq-\bar{\mu}_{1}\left(\left|z_{1 i}\right|^{\alpha+1}+\left|z_{2 i}\right|^{\alpha+1}\right)-\bar{\mu}_{2}\left(z_{1 i}^{2}+z_{2 i}^{2}\right)+\varrho\|\varepsilon\| \\
\dot{V}_{1} \leq-\bar{\mu}_{1} 2^{(\alpha+1) / 2} V_{1}^{(\alpha+1) / 2}-2 \bar{\mu}_{2} V_{1}+\sqrt{2} \varrho \sqrt{V_{1}} .
\end{array}
$$

Letting $\epsilon_{1}=\bar{\mu}_{1} 2^{(\alpha+1) / 2}$ and $\epsilon_{2}=2 \bar{\mu}_{2}$, we can change (32) into the following forms:

$$
\begin{aligned}
& \dot{V}_{1} \leq-\left(\epsilon_{2}-\frac{\sqrt{2} \varrho}{\sqrt{V_{1}}}\right) V_{1}-\epsilon_{1} V_{1}^{(\alpha+1) / 2}, \\
& \dot{V}_{1} \leq-\epsilon_{2} V_{1}-\left(\epsilon_{1}-\frac{\sqrt{2} \varrho}{V_{1}^{\alpha / 2}}\right) V_{1}^{(\alpha+1) / 2} .
\end{aligned}
$$

From (33), if $\epsilon_{2}-\sqrt{2} \varrho / \sqrt{V_{1}}>0$ then the finite-time stability is still ensured, and hence, by Lemma 5 , the state trajectories $z_{1 i}$ and $z_{2 i}$ of the system (28) converge to the region

$$
\|\varepsilon\| \leq \frac{2 \varrho}{\epsilon_{2}}
$$

in finite time. From (33), if $\epsilon_{1}-\sqrt{2} \varrho / V_{1}^{\alpha / 2}>0$, then the finite-time stability is still ensured, and hence, by Lemma 5 , the state trajectories $z_{1 i}$ and $z_{2 i}$ of the system (28) converge to the region

$$
\|\varepsilon\| \leq \sqrt{2}\left(\frac{\sqrt{2} \varrho}{\epsilon_{1}}\right)^{1 / \alpha}
$$


in finite time. Therefore, the state trajectories $z_{1 i}$ and $z_{2 i}$ of the system (28) converge in finite-time to the region

$$
\|\varepsilon\| \leq \Delta=\min \left\{\Delta_{1}, \Delta_{2}\right\}
$$

where $\Delta_{1}=2 \varrho / \epsilon_{2}$ and $\Delta_{2}=\sqrt{2}\left(\sqrt{2} \varrho / \epsilon_{1}\right)^{1 / \alpha}$.

Remark 8. The accuracy of tracking errors $z_{1 i}$ and $z_{2 i}$ is determined by the control parameters $\mu_{1 i}, \mu_{2 i}, \mu_{3 i}$, and $\mu_{4 i}$, $i=1,2,3$. To obtain the desired accuracy, it is necessary to reduce the size of the region (37) by increasing the values of the control parameters $\mu_{1 i}, \mu_{2 i}, \mu_{3 i}$, and $\mu_{4 i}$. However, using large values of these parameters leads to high magnitudes of control torques.

\section{Adaptive-Gain SOSMC Algorithm}

Next, the proposed adaptive-gain SOSMC scheme is designed by modifying the smooth SOSMC law presented in [27]. In stead of using the constant controller gains as presented in [27], the adaptive gains are considered in this paper. A novel adaptive law to tune these gains is presented. The main advantages of the proposed method are that only one parameter has to be tuned and this method relaxes the requirement of prior knowledge of the bound of system uncertainties.

The proposed control law is given by

$$
\begin{aligned}
u=B_{0}^{-1}(- & -\kappa-\kappa x_{2}-\lambda(t) \operatorname{sign}^{(p-1) / p}(S)-\epsilon(t) S \\
& -\alpha(t) \int_{0}^{t} \operatorname{sign}^{(p-2) / p}(S(\tau)) d \tau \\
& \left.-K(t) \int_{0}^{t} S(\tau) d \tau\right),
\end{aligned}
$$

where $p>2$ and the adaptive gains $\lambda(t), \alpha(t), \epsilon(t)$, and $K(t)$ are the design parameters defined as

$$
\begin{array}{r}
\lambda(t)=\lambda_{0} \operatorname{diag}\left(L^{(p-1) / p}(t), L^{(p-1) / p}(t), L^{(p-1) / p}(t)\right), \\
\alpha(t)=\alpha_{0} \operatorname{diag}(L(t), L(t), L(t)), \\
\epsilon(t)=\epsilon_{0} \operatorname{diag}(L(t), L(t), L(t)), \\
K(t)=K_{0} \operatorname{diag}\left(L^{2}(t), L^{2}(t), L^{2}(t)\right)
\end{array}
$$

with $\lambda_{0}, \alpha_{0}, \epsilon_{0}, K_{0}$ being positive constants. The adaptive law of the time-varying function $L(t)$ is given by

$$
\dot{L}(t)= \begin{cases}l, & \text { for }\left|S_{i}\right| \neq 0, \\ 0, & \text { for }\left|S_{i}\right|=0,\end{cases}
$$

where $l$ and the initial value $L(0)$ are positive constants.

Substituting (38) into (14), we obtain

$$
\begin{aligned}
\dot{S}= & -\lambda(t) \operatorname{sign}^{(p-1) / p}(S)-\epsilon(t) S-\alpha(t) \\
& \times \int_{0}^{t} \operatorname{sign}^{(p-2) / p} S(\tau) d \tau-K(t) \int_{0}^{t} S(\tau) d \tau+\tilde{d} .
\end{aligned}
$$

Next, the same procedure as presented in Section 3 is recalled. We can have the scalar form of (41) as

$$
\begin{array}{r}
\dot{z}_{1 i}=-\lambda(t)\left|z_{1 i}\right|^{(p-1) / p} \operatorname{sign}\left(z_{1 i}\right)-\epsilon(t) z_{1 i}+z_{2 i}, \\
\dot{z}_{2 i}=-\alpha(t)\left|z_{1 i}\right|^{(p-2) / p} \operatorname{sign}\left(z_{1 i}\right)-K(t) z_{1 i}+\delta_{i}, \\
i=1,2,3 .
\end{array}
$$

Theorem 9. Suppose that $\lambda_{0}, \alpha_{0}, \epsilon_{0}, K_{0}>0$ are selected such that

$$
\alpha_{0} K_{0}>\left[\frac{\alpha_{0} p}{p-1}+\left(\frac{2 p-1}{p}\right)^{2} \lambda_{0}^{2}\right] \epsilon_{0}^{2},
$$

and Assumptions 1 and 2 hold. Then, all trajectories of the system (42) converge in finite time to the region

$$
\Omega=\left\{\|\xi\| \leq\left(\frac{\gamma_{3}}{L(t) \gamma_{1}}\right)^{(p-1) /(p-2)}\right\},
$$

where $\xi=\left[L^{(p-1) / p}\left|z_{1 i}\right|^{(p-1) / p} \operatorname{sign}\left(z_{1 i}\right) L z_{1 i} z_{2 i}\right]^{T}$ and the positive scalars $\gamma_{1}$ and $\gamma_{3}$ will be defined later.

Proof. Let the Lyapunov function be chosen as

$$
\begin{aligned}
V_{2}(z)= & \frac{\alpha_{0} p}{p-1} L^{2(p-1) / p}\left|z_{1 i}\right|^{2(p-1) / p}+K_{0} L^{2} z_{1 i}^{2}+\frac{1}{2} z_{2 i}^{2} \\
& +\frac{1}{2}\left(\lambda_{0} L^{(p-1) / p}\left|z_{1 i}\right|^{(p-1) / p} \operatorname{sign}\left(z_{1 i}\right)+\epsilon_{0} L z_{1 i}-z_{2 i}\right)^{2} .
\end{aligned}
$$

The selected Lyapunov function can be expressed as

$$
\begin{aligned}
V_{2}(z)= & \left(\frac{\alpha_{0} p}{p-1}+\frac{1}{2} \lambda_{0}^{2}\right) L^{2(p-1) / p}\left|z_{1 i}\right|^{2(p-1) / p}+z_{2 i}^{2} \\
& +\left(K_{0}+\frac{1}{2} \epsilon_{0}^{2}\right) L^{2} z_{1 i}^{2}+\lambda_{0} \epsilon_{0}\left|z_{1 i}\right| L^{(p-1) / p}\left|z_{1 i}\right|^{(p-1) / p} \\
& -\lambda_{0} L^{(p-1) / p}\left|z_{1 i}\right|^{(p-1) / p} \operatorname{sign}\left(z_{1 i}\right) z_{2 i}-\epsilon_{0} L z_{1 i} z_{2 i},
\end{aligned}
$$

which can be written as

$$
V_{2}(z)=\xi^{T} \Pi_{1} \xi
$$

where

$$
\Pi_{1}=\frac{1}{2}\left[\begin{array}{ccc}
\frac{2 \alpha_{0} p}{p-1}+\lambda_{0}^{2} & \lambda_{0} \epsilon_{0} & -\lambda_{0} \\
\lambda_{0} \epsilon_{0} & \left(2 K_{0}+\epsilon_{0}^{2}\right) & -\epsilon_{0} \\
-\lambda_{0} & -\epsilon_{0} & 2
\end{array}\right] .
$$

It satisfies

$$
\sigma_{\min }\left(\Pi_{1}\right)\|\xi\|^{2} \leq V_{2} \leq \sigma_{\max }\left(\Pi_{1}\right)\|\xi\|^{2}
$$


where $\|\xi\|^{2}=L^{2(p-1) / p}\left|z_{1 i}\right|^{2(p-1) / p}+L^{2} z_{1 i}^{2}+z_{2 i}^{2}$. The time derivative of $V_{2}$ is obtained as

$$
\begin{aligned}
\dot{V}_{2} \leq & \frac{1}{2}\left(4 \alpha_{0}+\lambda_{0}^{2} \frac{p-1}{p}\right) L^{(2 p-2) / p}\left|z_{1 i}\right|^{(p-2) / p} \operatorname{sign}\left(z_{1 i}\right) \dot{z}_{1 i} \\
& +2 z_{2 i} \dot{z}_{2 i}+\left(2 K_{0}+\epsilon_{0}^{2}\right) L^{2} z_{1 i} \dot{z}_{1 i} \\
& +\lambda_{0} \epsilon_{0}\left(\frac{2 p-1}{p}\right) L^{(p-1) / p}\left|z_{1 i}\right|^{(p-1) / p} \operatorname{sign}\left(z_{1 i}\right) \dot{z}_{1 i} \\
& -\lambda_{0} L^{(p-1) / p}\left|z_{1 i}\right|^{-1 / p} z_{1 i} \dot{z}_{2 i} \\
& -\lambda_{0} L^{(p-1) / p}\left(\frac{p-1}{p}\right)\left|z_{1 i}\right|^{-1 / p} z_{2 i} \dot{z}_{1 i} \\
& -\epsilon_{0} L z_{1 i} \dot{z}_{2 i}-\epsilon_{0} L z_{2 i} \dot{z}_{1 i} \\
& +\frac{1}{2}\left(4 \alpha_{0}+\lambda_{0}^{2} \frac{p-1}{p}\right)\left|z_{1 i}\right|^{2(p-1) / p} L^{(p-2) / p} \dot{L} \\
& +\left(2 K_{0}+\epsilon_{0}^{2}\right) L \dot{L} z_{1 i}^{2} \\
& +\lambda_{0} \epsilon_{0}\left(\frac{(2 p-1)}{p}\right) L^{(p-1) / p}\left|z_{1 i}\right|^{(p-1) / p} \dot{L} \\
& +\lambda_{0} L^{(p-1) / p}\left|z_{1 i}\right|^{(p-1) / p} \operatorname{sign}\left(z_{1 i}\right) z_{2 i} L^{-1 / p} \dot{L} \\
& -\epsilon_{0} z_{1 i} z_{2 i} \dot{L} .
\end{aligned}
$$

Substituting $\dot{z}_{1 i}$ and $\dot{z}_{2 i}$ into (50), we obtain

$$
\begin{aligned}
\dot{V}_{2} \leq & \frac{1}{2}\left(4 \alpha_{0}+\lambda_{0}^{2} \frac{p-1}{p}\right) L^{(2 p-2) / p}\left|z_{1 i}\right|^{(p-2) / p} \operatorname{sign}\left(z_{1 i}\right) \\
& \times\left(z_{2 i}-\lambda_{0} L^{(p-1) / p}\left|z_{1 i}\right|^{(p-1) / p} \operatorname{sign}\left(z_{1 i}\right)-\epsilon_{0} L z_{1 i}\right) \\
& +2 z_{2 i}\left(-\alpha_{0} L\left|z_{1 i}\right|^{(p-2) / p} \operatorname{sign}\left(z_{1 i}\right)\right. \\
& \left.\quad-K_{0} L^{2} z_{1 i}+\delta_{i}\right) \\
& +\left(2 K_{0}+\epsilon_{0}^{2}\right) L^{2} z_{1 i} \\
& \times\left(-\lambda_{0} L^{(p-1) / p}\left|z_{1 i}\right|^{(p-1) / p} \operatorname{sign}\left(z_{1 i}\right)\right. \\
& \left.\quad-\epsilon_{0} L z_{1 i}+z_{2 i}\right) \\
+ & \left(\frac{2 p-1}{p}\right) \lambda_{0} \epsilon_{0} L^{(2 p-1) / p}\left|z_{1 i}\right|^{(p-1) / p} \operatorname{sign}\left(z_{1 i}\right) \\
& \times\left(-\lambda_{0} L^{(p-1) / p}\left|z_{1 i}\right|^{(p-1) / p} \operatorname{sign}\left(z_{1 i}\right)\right. \\
& \left.\quad-\epsilon_{0} L z_{1 i}+z_{2 i}\right) \\
& -\lambda_{0} L^{(p-1) / p}\left|z_{1 i}\right|^{-1 / p} z_{1 i}
\end{aligned}
$$

$$
\begin{aligned}
& \times\left(-\alpha_{0} L\left|z_{1 i}\right|^{(p-2) / p} \operatorname{sign}\left(z_{1 i}\right)-K_{0} L^{2} z_{1 i}+\delta_{i}\right) \\
& -\lambda_{0} L^{(p-1) / p}\left(\frac{p-1}{p}\right)\left|z_{1 i}\right|^{-1 / p} z_{2 i} \\
& \times\left(-\lambda_{0} L^{(p-1) / p}\left|z_{1 i}\right|^{(p-1) / p} \operatorname{sign}\left(z_{1 i}\right)\right. \\
& \left.-\epsilon_{0} L z_{1 i}+z_{2 i}\right) \\
& -\epsilon_{0} L z_{1 i}\left(-\alpha_{0} L\left|z_{1 i}\right|^{(p-2) / p} \operatorname{sign}\left(z_{1 i}\right)\right. \\
& -\epsilon_{0} L z_{2 i}\left(-\lambda_{0} L^{(p-1) / p}\left|z_{1 i}\right|^{(p-1) / p} \operatorname{sign}\left(z_{1 i}\right)\right. \\
& \left.\quad-\epsilon_{0} L z_{1 i}+z_{2 i}\right) \\
& +\frac{1}{2}\left(4 \alpha_{0}+\lambda_{0}^{2} \frac{p-1}{p}\right)\left|z_{1 i}\right|^{2(p-1) / p} L^{(p-2) / p} \dot{L} \\
& +\left(2 K_{0}+\epsilon_{0}^{2}\right) L \dot{L} z_{1 i}^{2} \\
& +\lambda_{0} \epsilon_{0}\left(\frac{(2 p-1)}{p}\right) L^{-1 / p}\left|z_{1 i}\right|^{(p-1) / p} \dot{L} \\
& +\epsilon_{0} \frac{p-1}{p} \mid z_{1 i} z_{2 i} \dot{L}^{(p-1) / p} \operatorname{sign}\left(z_{1 i}\right) z_{2 i} L^{-1 / p} \dot{L} \\
& +
\end{aligned}
$$

After lengthy algebraic manipulation, one obtains

$$
\begin{gathered}
\dot{V}_{2} \leq\left|z_{1 i}\right|^{-1 / p} L^{-1 / p} \\
\times\left(\left[\alpha_{0} \lambda_{0}+\lambda_{0}^{3} \frac{(p-1)}{p}\right]\left|z_{1 i}\right|^{2(p-1) / p} L^{2(p-1) / p}\right. \\
-2 \lambda_{0}^{2} \frac{(p-1)}{p} L^{(p-1) / p}\left|z_{1 i}\right|^{(p-1) / p} \operatorname{sign}\left(z_{1 i}\right) z_{2 i} \\
+\left[\lambda_{0} K_{0}+\frac{1}{2} \lambda_{0} \epsilon_{0}^{2} \frac{(6 p-2)}{p}\right] z_{1 i}^{2} L^{2} \\
\left.\quad-\lambda_{0} \epsilon_{0} \frac{(4 p-2)}{p} L z_{1 i} z_{2 i}+\frac{(p-1)}{p} \lambda_{0} z_{2 i}^{2}\right) \\
-\left(\left[\epsilon_{0} \alpha_{0}+\epsilon_{0}^{3} \frac{(3 p-2)}{p}\right]\left|z_{1 i}\right|^{2(p-1) / p} L^{2(p-1) / p}\right. \\
\left.+\left(\epsilon_{0} K_{0}+\epsilon_{0}^{3}\right) z_{1 i}^{2} L^{2}+2 \epsilon_{0}^{2} L z_{1 i} z_{2 i}-\epsilon_{0} z_{2 i}^{2}\right)
\end{gathered}
$$




$$
\begin{aligned}
& -\delta_{i}\left|z_{1 i}\right|^{(p-1) / p} L^{(p-1) / p} \operatorname{sign}\left(z_{1 i}\right)-\delta_{i} \epsilon_{0} L z_{1 i}+2 \delta_{i} z_{2 i} \\
& +\frac{\dot{L}(t)}{L}\left[\left(2 \alpha_{0}+\lambda_{0}^{2} \frac{p-1}{p}\right) L^{(2 p-2) / p}\left|z_{1 i}\right|^{(2 p-2) / p}\right] \\
& +\left(2 K_{0}+\epsilon_{0}^{2}\right) L^{2} \dot{L} z_{1 i}^{2}+\lambda_{0} \epsilon_{0} \frac{2 p-1}{p}\left|z_{1 i}\right|^{(2 p-1) / p} L^{(p-1) / p} \dot{L} \\
& -\lambda_{0} \frac{p-1}{p} L^{(p-1) / p}\left|z_{1 i}\right|^{(p-1) / p} \operatorname{sign}\left(z_{1 i}\right) z_{2 i}-\epsilon_{0} L z_{1 i} z_{2 i},
\end{aligned}
$$

which can be written as

$$
\begin{aligned}
\dot{V}_{2}= & -\left|z_{1 i}\right|^{-1 / p} L^{-1 / p} L(t) \xi^{T} \Omega_{1} \xi \\
& -L(t) \xi^{T} \Omega_{2} \xi+\delta_{i} \Gamma_{1} \xi+\frac{\dot{L}(t)}{L} \Gamma_{2} \Pi_{2} \xi,
\end{aligned}
$$

where $\quad \Gamma_{1} \quad=\quad\left[\begin{array}{lll}-\lambda_{0} & -\epsilon_{0} & 2\end{array}\right]^{T}, \quad \Gamma_{2}=$ $\left[\begin{array}{lll}L^{(p-1) / p}\left|z_{1 i}\right|^{(p-1) / p} \operatorname{sign}\left(z_{1 i}\right) & L z_{1 i} & 0\end{array}\right]^{T}$,

$$
\begin{aligned}
& \Omega_{1}=\frac{\lambda_{0}}{2} \\
& \times\left[\begin{array}{ccc}
2 \alpha_{0}+\lambda_{0}^{2}\left(\frac{2 p-2}{p}\right) & 0 & -\lambda_{0}\left(\frac{2 p-2}{p}\right) \\
0 & 2 K_{0}+\epsilon_{0}^{2}\left(\frac{6 p-2}{p}\right) & -\epsilon_{0}\left(\frac{4 p-2}{p}\right) \\
-\lambda_{0}\left(\frac{2 p-2}{p}\right) & -\epsilon_{0}\left(\frac{4 p-2}{p}\right) & \frac{2(p-1)}{p}
\end{array}\right], \\
& \Omega_{2}=\epsilon_{0}\left[\begin{array}{ccc}
\alpha_{0}+\lambda_{0}^{2}\left(\frac{3 p-2}{p}\right) & 0 & 0 \\
0 & K_{0}+\epsilon_{0} & -\epsilon_{0} \\
0 & -\epsilon_{0} & 1
\end{array}\right] \text {, } \\
& \Pi_{2}=\frac{1}{2}\left[\begin{array}{ccc}
4 \alpha_{0}+2 \lambda_{0}^{2}\left(\frac{p-1}{p}\right) & \lambda_{0} \epsilon_{0}\left(\frac{2 p-1}{p}\right) & -\lambda_{0} \\
\lambda_{0} \epsilon_{0}\left(\frac{2 p-1}{p}\right) & \left(2 K_{0}+\epsilon_{0}^{2}\right) & -\epsilon_{0} \\
-\lambda_{0} & -\epsilon_{0} & 2
\end{array}\right] .
\end{aligned}
$$

By Assumption 2 and using $\|\xi\| \geq\left\|\Gamma_{2}\right\|$, one obtains

$$
\begin{aligned}
\dot{V}_{2}= & -\left|z_{1 i}\right|^{-1 / p} L^{-1 / p} L(t) \sigma_{\min }\left(\Omega_{1}\right)\|\xi\|^{2} \\
& -L(t) \sigma_{\min }\left(\Omega_{2}\right)\|\xi\|^{2}+\left|\delta_{i}\right|\left\|\Gamma_{1}\right\|\|\xi\| \\
& +\frac{\dot{L}(t)}{L} \sigma_{\min }\left(\Pi_{2}\right)\|\xi\|^{2} .
\end{aligned}
$$

Using $\left|z_{1 i}\right|^{-1 / p} L^{-1 / p} \geq\|\xi\|^{-1 /(p-1)}$, we have

$$
\begin{aligned}
\dot{V}_{2}= & -L(t) \sigma_{\min }\left(\Omega_{1}\right)\|\xi\|^{(2 p-3) /(p-1)} \\
& -L(t) \sigma_{\min }\left(\Omega_{2}\right)\|\xi\|^{2}+\varrho\left\|\Gamma_{1}\right\|\|\xi\| \\
& +\frac{\dot{L}(t)}{L} \sigma_{\max }\left(\Pi_{2}\right)\|\xi\|^{2} .
\end{aligned}
$$

Using (49), the expression (56) becomes

$$
\begin{aligned}
\dot{V}_{2}= & -L(t) \frac{\sigma_{\min }\left(\Omega_{1}\right)}{\sqrt{\sigma_{\max }\left(\Pi_{1}\right)}}\|\xi\|^{(p-2) /(p-1)} V_{2}^{1 / 2} \\
& -L(t) \frac{\sigma_{\min }\left(\Omega_{2}\right)}{\sigma_{\max }\left(\Pi_{1}\right)} V_{2}+\varrho \frac{\left\|\Gamma_{1}\right\|}{\sqrt{\sigma_{\min }\left(\Pi_{1}\right)}} V_{2}^{1 / 2} \\
& +\frac{\dot{L}(t)}{2 L(t)} \frac{\sigma_{\max }\left(\Pi_{2}\right)}{\sigma_{\min }\left(\Pi_{1}\right)} V_{2} .
\end{aligned}
$$

From simplicity, we define

$$
\begin{array}{ll}
\gamma_{1}=\frac{\sigma_{\min }\left(\Omega_{1}\right)}{\sqrt{\sigma_{\max }\left(\Pi_{1}\right)}}, & \gamma_{2}=\frac{\left\|\sigma_{\min }\left(\Omega_{2}\right)\right\|}{\sigma_{\max }\left(\Pi_{1}\right)}, \\
\gamma_{3}=\varrho \frac{\left\|\Gamma_{1}\right\|}{\sqrt{\sigma_{\min }\left(\Pi_{1}\right)}}, & \gamma_{4}=\frac{\sigma_{\max }\left(\Pi_{2}\right)}{2 \sigma_{\min }\left(\Pi_{1}\right)},
\end{array}
$$

where $\gamma_{1}, \gamma_{2}, \gamma_{3}$, and $\gamma_{4}$ are positive constants. Thus, (57) can be written as

$$
\begin{aligned}
\dot{V}_{2} \leq & -\left(L(t) \gamma_{1}\|\xi\|^{(p-2) /(p-1)}-\gamma_{3}\right) V_{2}^{1 / 2} \\
& -\left(L(t) \gamma_{2}-\frac{\dot{L}(t)}{L} \gamma_{4}\right) V_{2} .
\end{aligned}
$$

Because $\dot{L}(t) \geq 0$ such that the term $L(t) \gamma_{2}-(\dot{L}(t) / L) \gamma_{4}$ is positive in finite time, it follows from (59) that, if $L(t) \gamma_{1}\|\xi\|^{(p-2) /(p-1)}>\gamma_{3}$, the error system (42) will converge in finite time to the region

$$
\Omega=\left\{\|\xi\| \leq\left(\frac{\gamma_{3}}{L(t) \gamma_{1}}\right)^{(p-1) /(p-2)}\right\} .
$$

In fact, we can choose $\lambda_{1 i}, \lambda_{2 i}, \lambda_{3 i}$, and $\lambda_{4 i}$ such that $\gamma_{3} / L(t) \gamma_{1}<1$. With $p>2,(p-1) /(p-2)$ being sufficiently large, so $\xi$ is sufficiently small in finite time.

Remark 10. The control law (38) includes an adaptive law which is used to compensate for the derivative of total disturbances $\widetilde{d}$. Normally, the bound of $\dot{\vec{d}}$ is much smaller than the bound of $\widetilde{d}$, so this supports the high accuracy of our proposed method.

\section{Simulation Results}

An example of attitude control of flexible spacecraft is presented with numerical simulations to demonstrate the performance of the developed controllers (25) and (38). The spacecraft is assumed to have the nominal inertia matrix [33]

$$
J=\left[\begin{array}{ccc}
350 & 3 & 4 \\
3 & 270 & 10 \\
4 & 10 & 190
\end{array}\right] \mathrm{kg} \cdot \mathrm{m}^{2}
$$




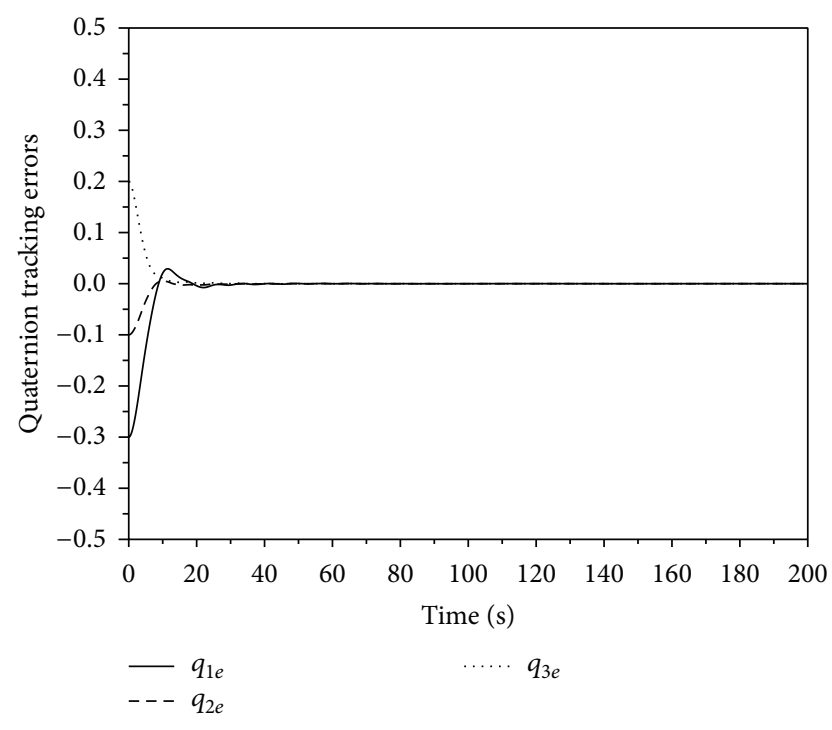

FIGURE 1: Quaternion tracking errors-controller (25).

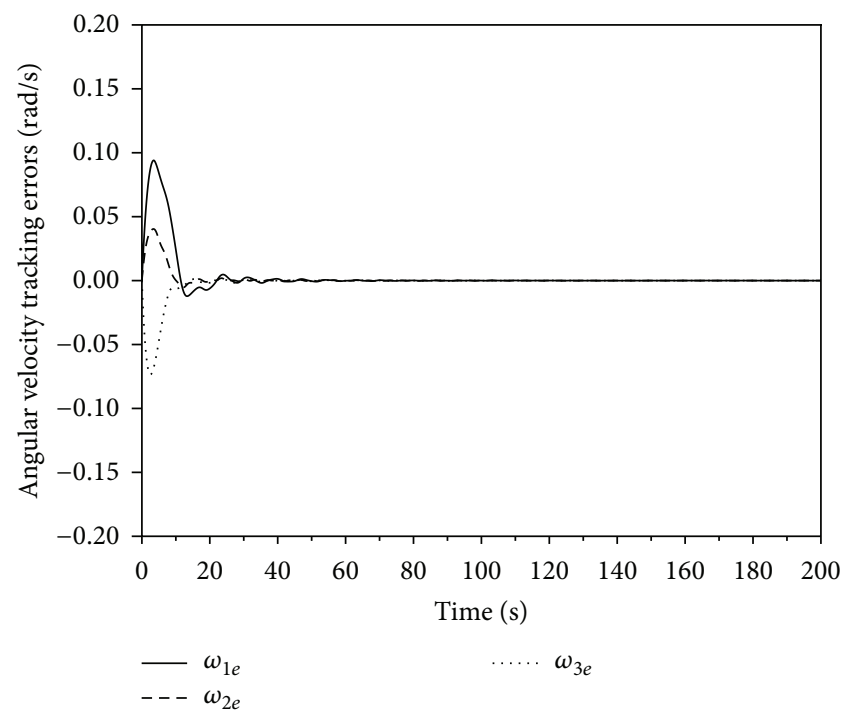

FIGURE 2: Angular velocity tracking errors-controller (25).

and coupling matrices

$$
\delta=\left[\begin{array}{ccc}
6.45637 & 1.27814 & 2.15629 \\
-1.25619 & 0.91756 & -1.67264 \\
1.11678 & 2.48901 & -0.83674 \\
1.23637 & -2.6581 & -1.12503
\end{array}\right] \mathrm{kg}^{1 / 2} \cdot \mathrm{m} / \mathrm{s}^{2},
$$

respectively. The first four elastic modes that have been considered in the model used for simulating a spacecraft are $\omega_{n 1}=0.7681, \omega_{n 2}=1.1038, \omega_{n 3}=1.8733$, and $\omega_{n 4}=$ $2.5496 \mathrm{rad} / \mathrm{sec}$ with damping $\xi_{1}=0.0056, \xi_{2}=0.0086$, $\xi_{3}=0.013$, and $\xi_{1}=0.025$. The initial states of the rotation motion are given by

$$
\begin{aligned}
& Q(0)=\left[\begin{array}{llll}
0.3 & -0.1 & 0.2 & 0.9274
\end{array}\right]^{T}, \\
& \omega(0)=0_{3 \times 1} \mathrm{rad} / \mathrm{sec}, \quad \vartheta(0)=0_{4 \times 1} \text {. }
\end{aligned}
$$

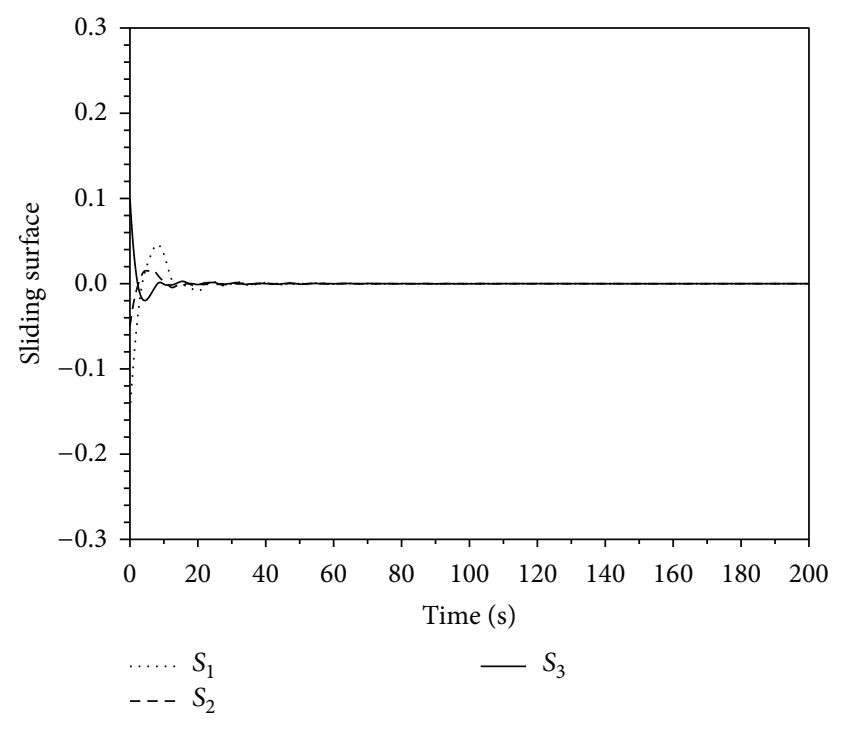

FIGURE 3: Switching function-controller (25).

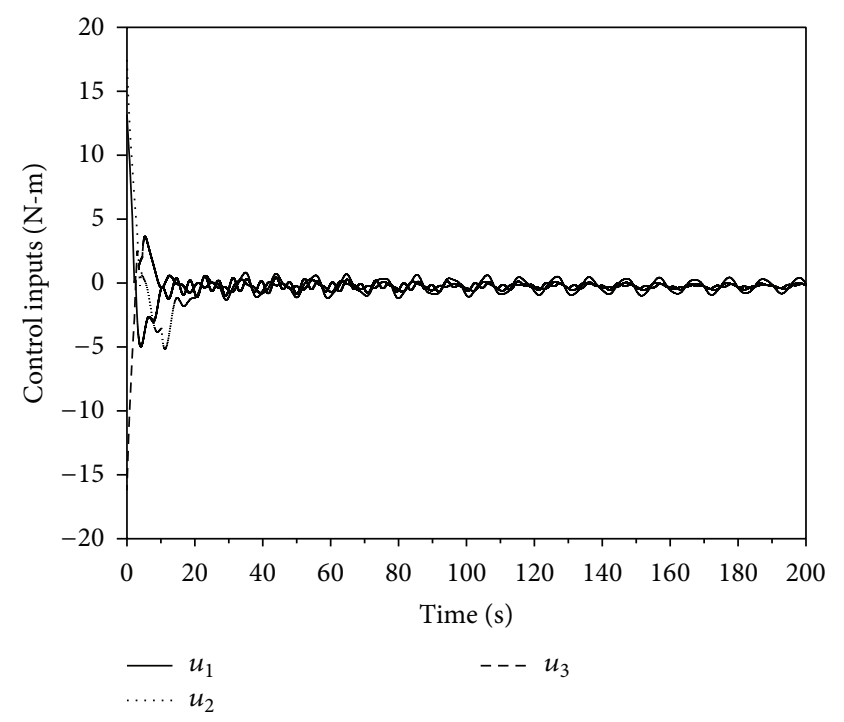

FIgURE 4: Control torques-controller (25).

For the controller (25), the chosen gains are given as $\mu_{1}=$ $\operatorname{diag}(15,15,15), \mu_{2}=\operatorname{diag}(10,10,10), \mu_{3}=\operatorname{diag}(5,5,5)$, and $\mu_{4}=\operatorname{diag}(10,10,10)$. On the other hand, for controller (38), the control gains are selected to be $\lambda_{0}=15, \alpha_{0}=10, \epsilon_{0}=10$, $K_{0}=15$, and $l=5 \times 10^{5}$. For both control algorithms, we use the same sliding manifold (23) with the constant $\kappa=1.2$.

The attitude control problem is considered in the presence of external disturbance $d(t)$. The external disturbances are described as

$$
d(t)=\left[\begin{array}{c}
0.3 \cos (0.1 t)+0.1 \\
0.15 \sin (0.1 t)+0.3 \cos (0.1 t) \\
0.3 \sin (0.1 t)+0.1
\end{array}\right] \mathrm{N}-\mathrm{m} .
$$




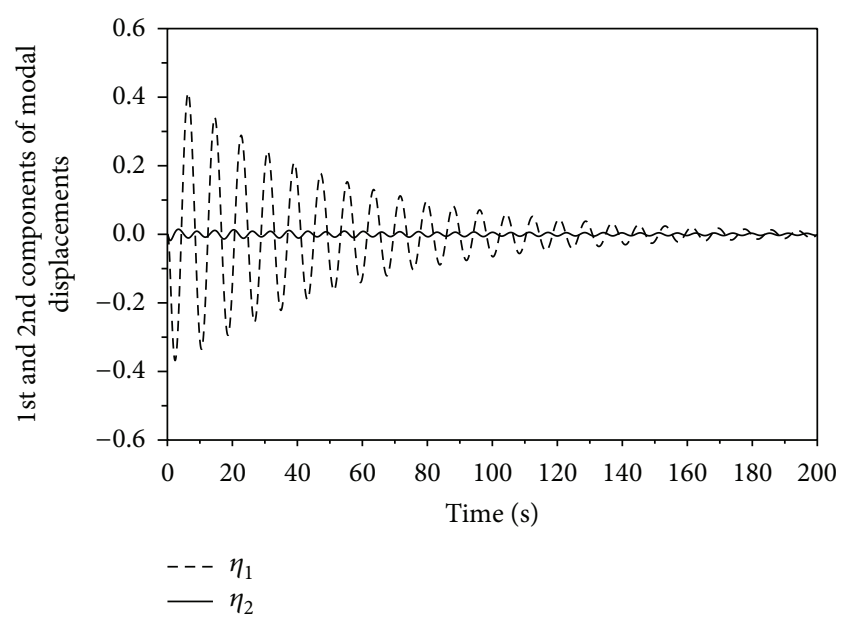

FIgURE 5: Modal displacements $\eta_{1}$ and $\eta_{2}$-controller (25).

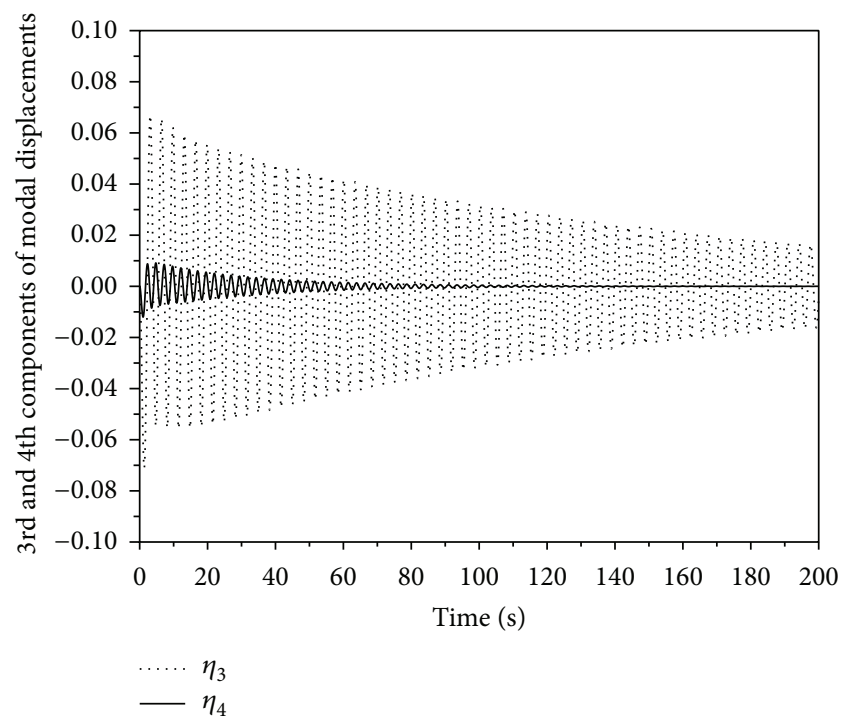

FIgURE 6: Modal displacements $\eta_{3}$ and $\eta_{4}$-controller (25).

And the desired angular velocity tracking is given by

$$
\omega_{d}(t)=\left[\begin{array}{c}
-0.04 \cos (0.2 t) \\
-0.04 \sin (0.2 t) \\
0.05 \sin (0.2 t)+\cos (0.2 t)
\end{array}\right] \mathrm{rad} / \mathrm{sec}
$$

together with

$$
q_{d}(0)=\left[\begin{array}{lll}
0 & 0 & 0
\end{array}\right]^{T} .
$$

For simulation results with controllers (25) and (38), the attitude quaternion tracking errors are shown in Figures 1 and 7 , and angular velocity tracking errors are illustrated in Figures 2 and 8. Controller (38) gives smoother attitude and angular velocity tracking outputs than controller (25). From Figures 3 and 9 , it can be seen that the sliding vectors are on the sliding surface $s=0$ after 40 seconds. The control torques of the controllers (38) and (25) are shown in Figures 4 and 10, respectively. It can be seen that controller (38) gives smoother

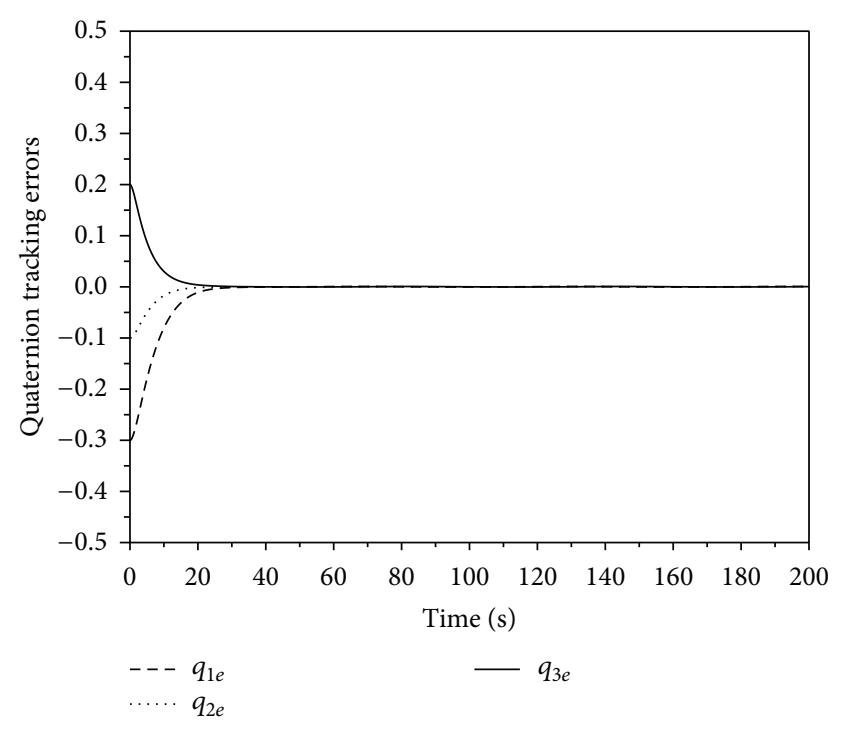

FIGURE 7: Quaternion tracking errors-controller (38).

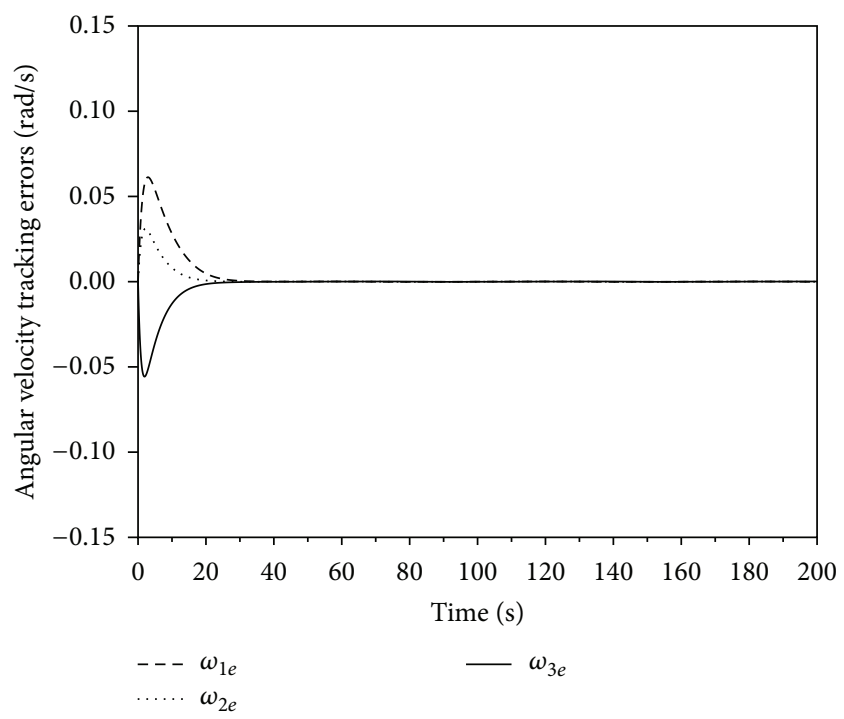

FIGURE 8: Angular velocity tracking errors-controller (38).

responses of control torques. With the larger magnitude, controller (38) makes the responses go to zero faster than controller (25), so it gives smoother responses during the first 40 seconds and this improves the transient performance. Thus, under the control law (38) the smoother velocity and attitude tracking error responses are obtained. The responses of modal displacements are presented in Figures 5, 6, 11, and, 12 , in which a low vibration level is illustrated for controller (38) in comparison with controller (25). For controller (25), the boundary layer $\|s\| \leq 7.63 \times 10^{-5}$ is achieved in finite time. Regarding the accuracy, the bounds on $\left\|q_{e}\right\|$ and $\left\|\omega_{e}\right\|$ are $\left\|q_{e}\right\| \leq 5.12 \times 10^{-5}$ and $\left\|\omega_{e}\right\| \leq 4.28 \times 10^{-5}$ with the sampling time $h=0.005$. Also, for controller (38), the boundary layer $\|s\| \leq 4.7 \times 10^{-5}$ is reached in finite time. Regarding the accuracy, the bounds on $\left\|q_{e}\right\|$ and $\left\|\omega_{e}\right\|$ are 


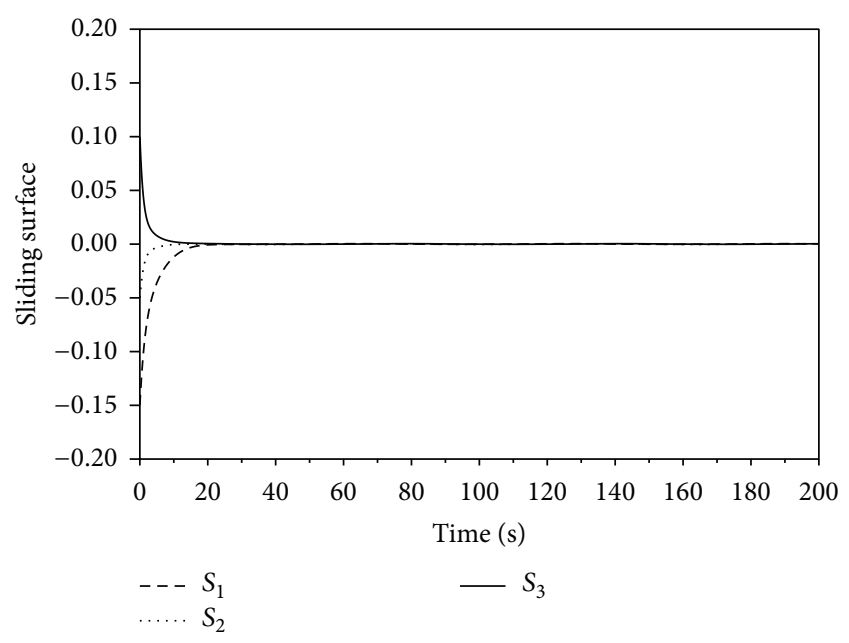

FIGURE 9: Switching function-controller (38).

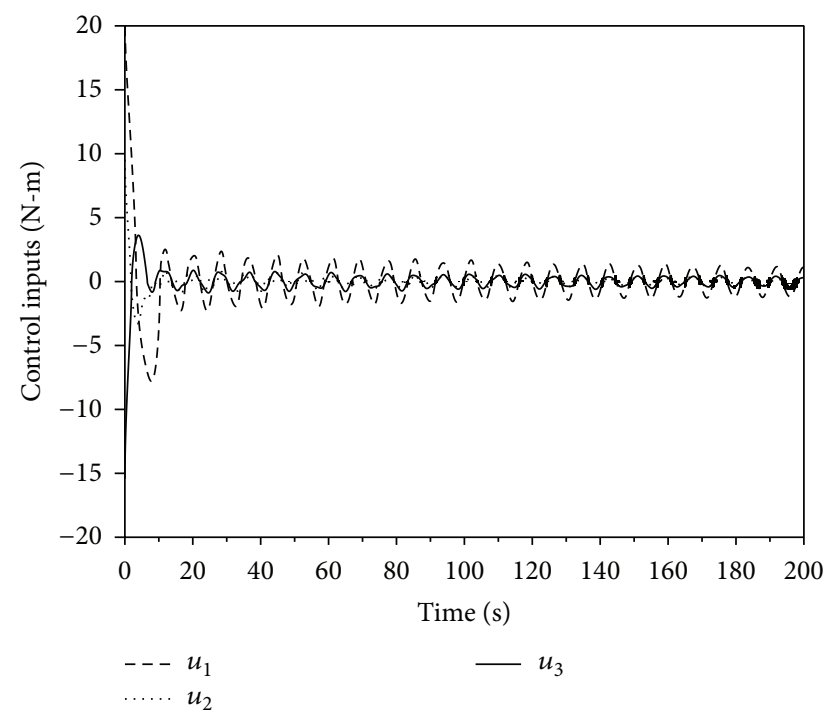

Figure 10: Control torques-controller (38).

$\left\|q_{e}\right\| \leq 9.35 \times 10^{-6}$ and $\left\|\omega_{e}\right\| \leq 1.5 \times 10^{-5}$ with the sampling time $h=0.005$.

A comparison of the simulation results obtained by (25) and (38) shows the following. Both control laws give high accuracy of attitude tracking outputs. Controller (38) provides smoother responses of attitude and angular velocity tracking errors when compared with controller (25). Moreover, controller (38) offers a lower vibration level of modal displacements. In view of these simulation results, controller (38) seems to give better performance for practical attitude tracking control of a flexible spacecraft.

\section{Conclusions}

The proposed finite-time controllers have been successfully applied to attitude tracking maneuvers of a flexible spacecraft. The first controller based on the novel SOSMC algorithm is

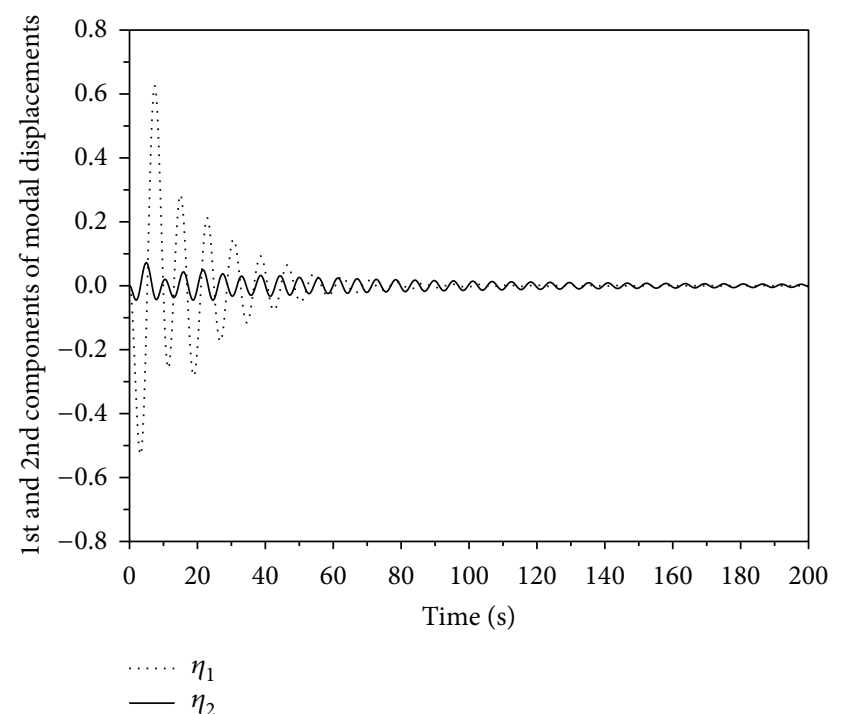

FIgURE 11: Modal displacements $\eta_{1}$ and $\eta_{2}$-controller (38).

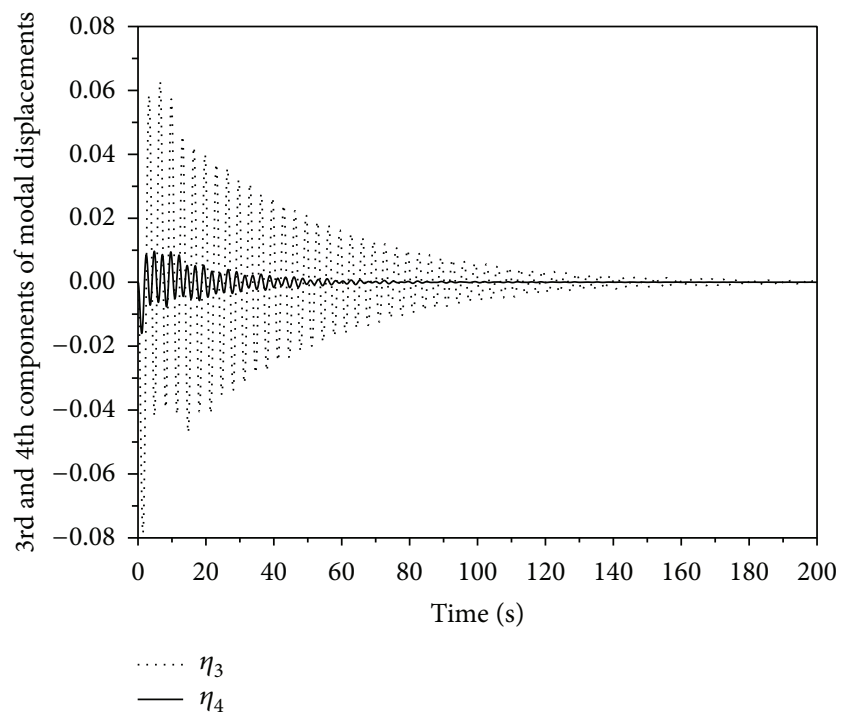

FIGURE 12: Modal displacements $\eta_{3}$ and $\eta_{4}$-controller (38).

developed to deal with quaternion-based spacecraft-attitudetracking maneuvers. For the second control law, an adaptivegain second-order sliding mode control algorithm is designed to relax the requirement of prior knowledge on the bound of system uncertainties. Both control laws achieve highprecision tracking performance and strong robustness ability. The concepts of the Lyapunov stability are employed to ensure a finite-time property of the proposed controllers. Numerical simulations on attitude tracking control are provided to demonstrate the performance of the developed controllers.

\section{Conflict of Interests}

The author declares that there is no conflict of interests regarding the publication of this paper. 


\section{Acknowledgment}

This research was supported by the National Research Council of Thailand.

\section{References}

[1] A. G. Kelkar, S. M. Joshi, and T. E. Alberts, "Dissipative controllers for nonlinear multibody flexible space systems," Journal of Guidance, Control, and Dynamics, vol. 18, no. 5, pp. 1044-1052, 1995.

[2] S. Di Gennaro, "Active vibration suppression in flexible spacecraft attitude tracking," Journal of Guidance, Control, and Dynamics, vol. 21, no. 3, pp. 400-408, 1998.

[3] Q. Hu, "Sliding mode attitude control with $L_{2}$-gain performance and vibration reduction of flexible spacecraft with actuator dynamics," Acta Astronautica, vol. 67, no. 5-6, pp. 572$583,2010$.

[4] B. Xiao, Q. Hu, and Y. Zhang, "Adaptive sliding mode fault tolerant attitude tracking control for flexible spacecraft under actuator saturation," IEEE Transactions on Control Systems Technology, vol. 20, no. 6, pp. 1605-1612, 2012.

[5] M. Shahravi, M. Kabganian, and A. Alasty, "Adaptive robust attitude control of a flexible spacecraft," International Journal of Robust and Nonlinear Control, vol. 16, no. 6, pp. 287-302, 2006.

[6] S. N. Singh and R. Zhang, "Adaptive output feedback control of spacecraft with flexible appendages by modeling error compensation," Acta Astronautica, vol. 54, no. 4, pp. 229-243, 2004.

[7] Q. Hu, "Variable structure maneuvering control with timevarying sliding surface and active vibration damping of flexible spacecraft with input saturation," Acta Astronautica, vol. 64, no. 11-12, pp. 1085-1108, 2009.

[8] Q. Hu and B. Xiao, "Fault-tolerant sliding mode attitude control for flexible spacecraft under loss of actuator effectiveness," Nonlinear Dynamics, vol. 64, no. 1-2, pp. 13-22, 2011.

[9] Y. Zeng, A. D. Araujo, and S. N. Singh, "Output feedback variable structure adaptive control of a flexible spacecraft," Acta Astronautica, vol. 44, no. 1, pp. 11-22, 1999.

[10] S. Di Gennaro, "Output attitude tracking for flexible spacecraft," Automatica, vol. 38, no. 10, pp. 1719-1726, 2002.

[11] J. Zheng, S. P. Banks, and H. Alleyne, "Optimal attitude control for three-axis stabilized flexible spacecraft," Acta Astronautica, vol. 56, no. 5, pp. 519-528, 2005.

[12] C. Pukdeboon, "Optimal sliding mode controllers for attitude stabilization of flexible spacecraft," Mathematical Problems in Engineering, vol. 2011, Article ID 863092, 20 pages, 2011.

[13] Q. Hu and B. Xiao, "Intelligent proportional-derivative control for flexible spacecraft attitude stabilization with unknown input saturation," Aerospace Science and Technology, vol. 23, no. 1, pp. 63-74, 2012.

[14] V. I. Utkin, Sliding Modes in Control and Optimization, Springer, Berlin, Germany, 1992.

[15] Q. Hu, P. Shi, and H. J. Gao, "Adaptive variable structure and commanding shaped vibration control of flexible spacecraft," Journal of Guidance, Control, and Dynamics, vol. 30, no. 3, pp. 804-815, 2007.

[16] J. Erdong and S. Zhaowei, "Robust attitude tracking control of flexible spacecraft for achieving globally asymptotic stability," International Journal of Robust and Nonlinear Control, vol. 19, no. 11, pp. 1201-1223, 2009.
[17] Z. Man, A. P. Paplinski, and H. R. Wu, "A robust MIMO terminal sliding mode control scheme for rigid robotic manipulators," IEEE Transactions on Automatic Control, vol. 39, no. 12, pp. 2464-2469, 1994.

[18] Y. Feng, X. Yu, and Z. Man, "Non-singular terminal sliding mode control of rigid manipulators," Automatica, vol. 38, no. 12, pp. 2159-2167, 2002.

[19] J. Erdong and S. Zhaowei, "Passivity-based control for a flexible spacecraft in the presence of disturbances," International Journal of Non-Linear Mechanics, vol. 45, no. 4, pp. 348-356, 2010.

[20] S. Wu, G. Radice, and Z. Sun, "Robust finite-time control for flexible spacecraft attitude maneuver," Journal of Aerospace Engineering, vol. 27, no. 1, pp. 185-190, 2014.

[21] W. Perruquetti and J. P. Barbot, Sliding Mode Control in Engineering, Marcel Dekker, New York, NY, USA, 2002.

[22] C. Edwards, E. Fossas Colet, and L. Fridman, Advances in Variable Structure and Sliding Mode Control, Lecture Notes in Control and Information Sciences, Springer, Berlin, Germany, 2006.

[23] A. Damiano, G. L. Gatto, I. Marongiu, and A. Pisano, "Secondorder sliding-mode control of dc drives," IEEE Transactions on Industrial Electronics, vol. 51, no. 2, pp. 364-373, 2004.

[24] G. Bartolini, A. Pisano, and E. Usai, "Second-order slidingmode control of container cranes," Automatica, vol. 38, no. 10, pp. 1783-1790, 2002.

[25] M. Canale, L. Fagiano, A. Ferrara, and C. Vecchio, "Vehicle yaw control via second-order sliding-mode technique," IEEE Transactions on Industrial Electronics, vol. 55, no. 11, pp. 39083916, 2008.

[26] C. Pukdeboon, A. S. I. Zinober, and M.-W. L. Thein, "Quasi-continuous higher order sliding-mode controllers for spacecraft-attitude-tracking maneuvers," IEEE Transactions on Industrial Electronics, vol. 57, no. 4, pp. 1436-1444, 2010.

[27] C. Pukdeboon, "Finite-time second-order sliding mode controllers for spacecraft attitude tracking," Mathematical Problems in Engineering, vol. 2012, Article ID 930269, 12 pages, 2013.

[28] W. Respondek, A. Pogromsky, and H. Nijmeijer, "Time scaling for observer design with linearizable error dynamics," Automatica, vol. 40, no. 2, pp. 277-285, 2004.

[29] J. R. Wertz, Spacecraft Attitude Determination and Control, Kluwer Academic Publishers, 1978.

[30] M. J. Sidi, Spacecraft Dynamics and Control, Cambridge University Press, Cambridge, UK, 1997.

[31] V. T. Haimo, "Finite time controllers," SIAM Journal on Control and Optimization, vol. 24, no. 4, pp. 760-771, 1986.

[32] S. P. Bhat and D. S. Bernstein, "Finite-time stability of continuous autonomous systems," SIAM Journal on Control and Optimization, vol. 38, no. 3, pp. 751-766, 2000.

[33] S. Di Gennaro, "Output stabilization of flexible spacecraft with active vibration suppression," IEEE Transactions on Aerospace and Electronic Systems, vol. 39, no. 3, pp. 747-759, 2003. 


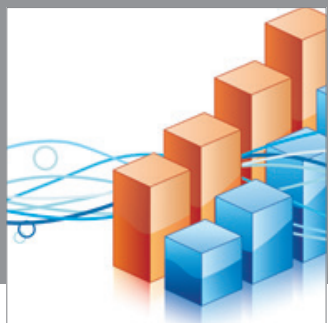

Advances in

Operations Research

mansans

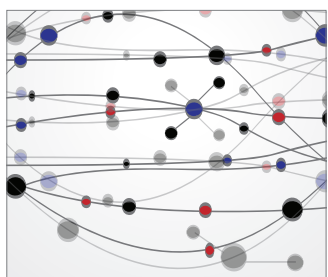

The Scientific World Journal
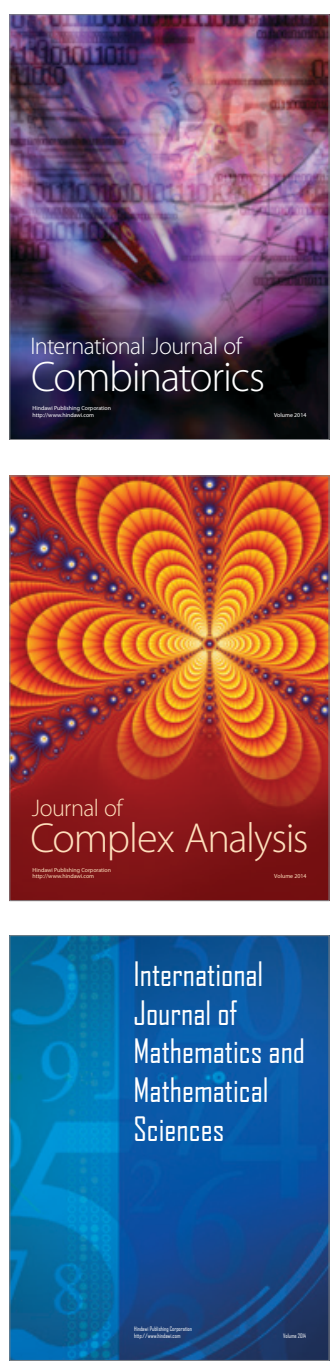
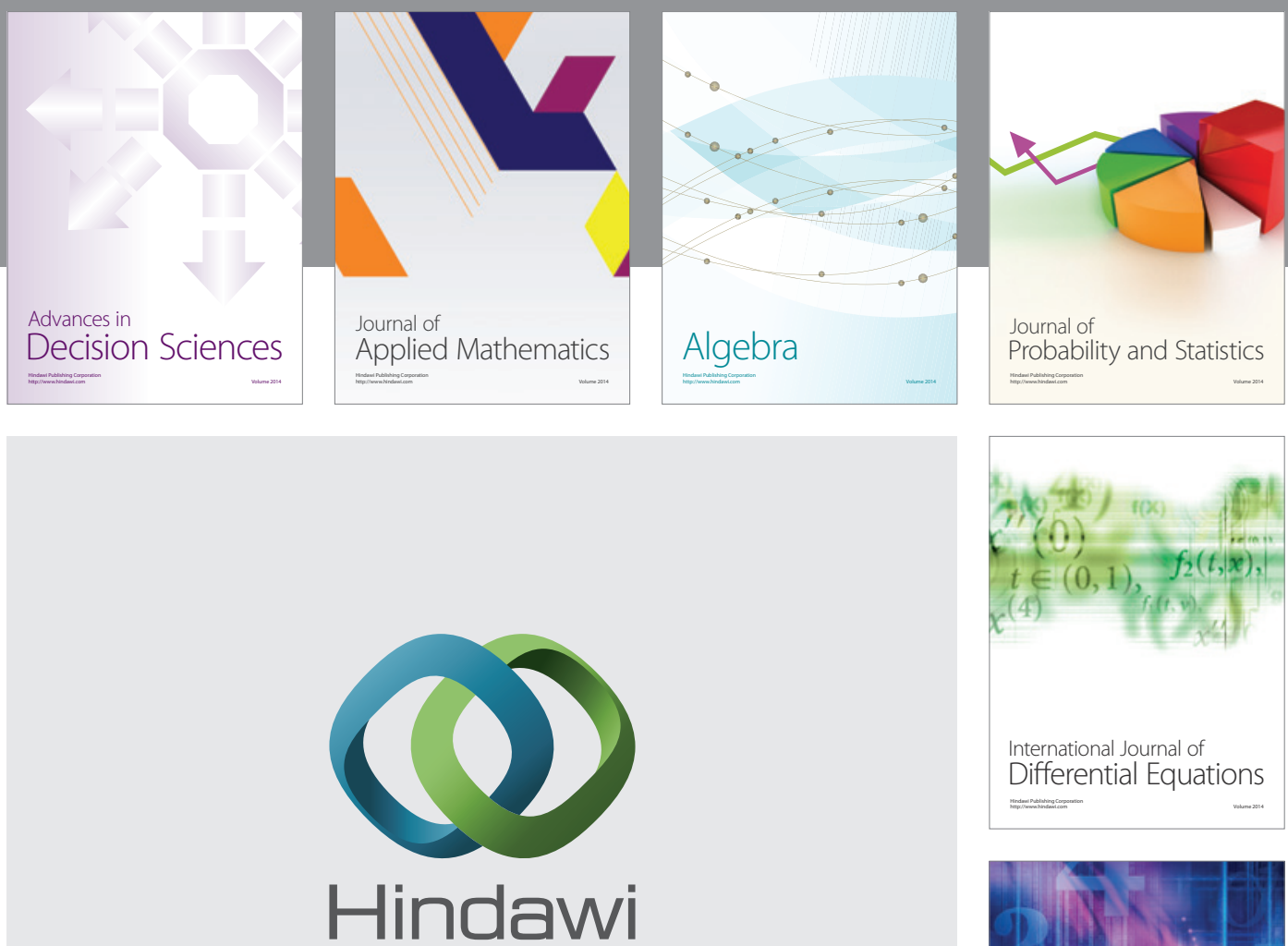

Submit your manuscripts at http://www.hindawi.com
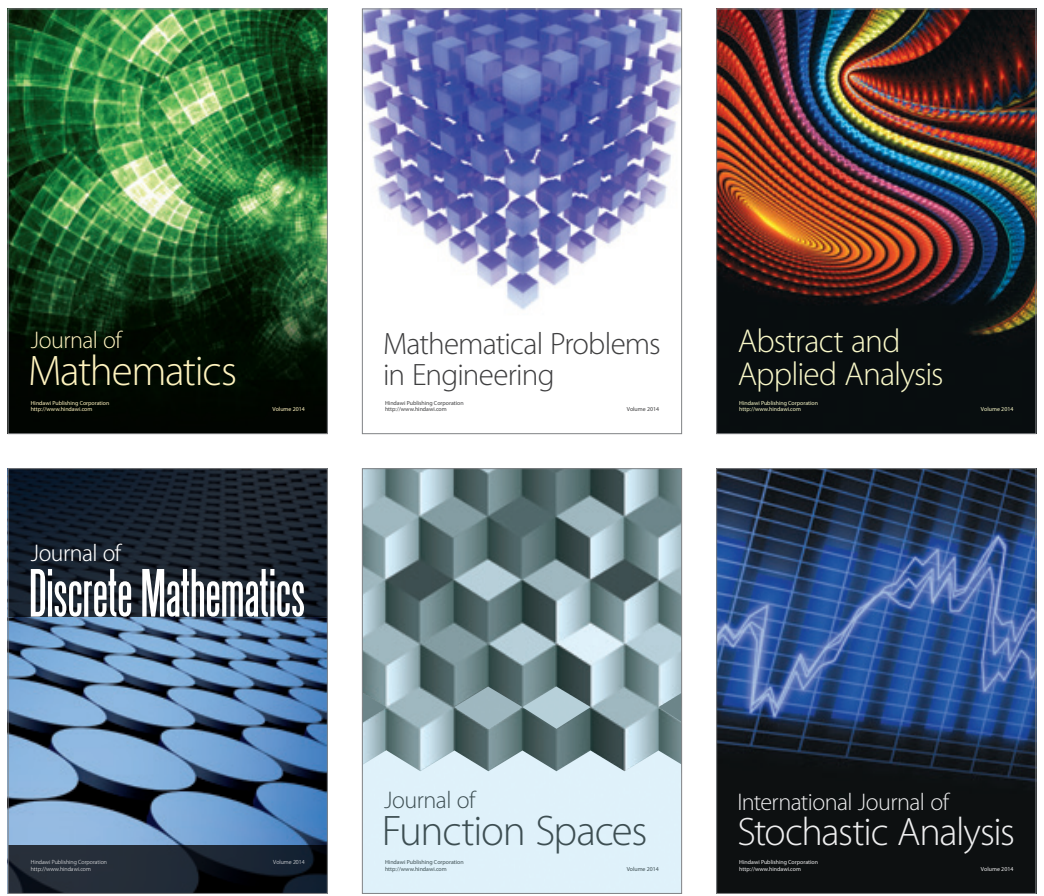

Journal of

Function Spaces

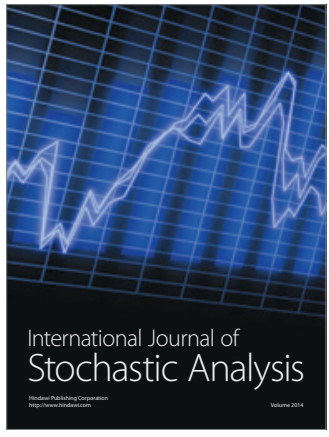

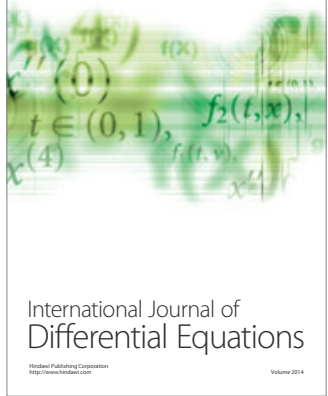
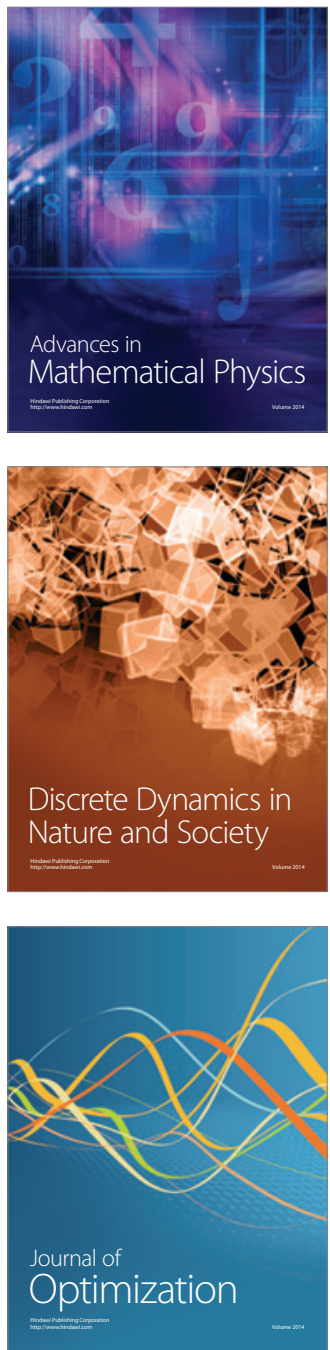